\title{
Low energy hadronic contribution to the QED vacuum polarization
}

\author{
H. Burkhardt* \\ CERN, AB Departement, CH-1211 Geneva 23, Switzerland \\ B. Pietrzyk ${ }^{\dagger}$ \\ Laboratoire de Physique des Particules LAPP, IN2P3-CNRS, F-74941 Annecy-le-Vieux Cedex, France
}

(Received 1 July 2005; published 7 September 2005)

\begin{abstract}
Recent improvements in the low energy $e^{+} e^{-}$annihilation data and their influence on the determination of the hadronic contribution to the running of the QED fine structure constant at $m_{Z}$ are discussed. Using CMD-2 and KLOE measurements in the $\rho$ region we obtain $\Delta \alpha_{\text {had }}^{(5)}(s)=0.02758 \pm 0.00035$ at $s=m_{Z}^{2}$.

DOI: 10.1103/PhysRevD.72.057501

PACS numbers: 13.85.Lg, 13.66.Jn, 12.15.Lk
\end{abstract}

In the year 2001, we published an updated evaluation of the hadronic contribution to the running of the QED fine structure constant [1], based on a dispersion integral using a parametrization of the measured cross section of $e^{+} e^{-} \rightarrow$ hadrons. We obtained a hadronic contribution of $\Delta \alpha_{\text {had }}^{(5)}(s)=0.02761 \pm 0.00036$ at $s=m_{Z}^{2}$.

Our parametrization in the c.m. energy region of the $\rho$, the contribution of the $\pi^{+} \pi^{-}$final state from threshold to $1.8 \mathrm{GeV}$, was based on a pion form factor parametrization obtained by the CMD-2 Collaboration which used results of their measurements in the c.m. energy region between 0.61 and $0.96 \mathrm{GeV}$ at the VEPP-2M collider [2]. The overall uncertainty of the $\rho$ region integral, including the statistical uncertainty, was $2.3 \%$ (that of $\Gamma_{e e}$ in [2]) in our analysis.

Since then, the CMD-2 Collaboration improved the treatment of radiative corrections twice. An intermediate improvement has appeared in the published document [3] and an additional improvement has become available in 2004 [4]. We have concluded that the most recent CMD-2 results imply only a small change in the estimate of the hadronic contribution [5].

Recently, the KLOE Collaboration [6] has measured the cross section of $e^{+} e^{-} \rightarrow \pi^{+} \pi^{-}$with high statistical accuracy in small energy bins using the "radiative return" from the $\phi$ resonance to the $\rho$ in the $\pi^{+} \pi^{-}$mass range between 0.59 and $0.97 \mathrm{GeV}$.

We have been repeatedly asked to update our previous analysis and to comment on and quantify the influence of recent low energy measurements by KLOE and CMD- 2 on our results. We find that the actual change turns out to be very small. Thus, we have decided to submit this work as a brief report. To the extent that this report is an update of a previously published article, the choice is not to unnecessarily repeat the discussion for energy regions which did not change.

In our 2001 analysis, we used the parametrization of the pion form factor obtained by the CMD-2 Collaboration.

\footnotetext{
*Electronic address: Helmut.Burkhardt@cern.ch

${ }^{\dagger}$ Electronic address: pietrzyk@lapp.in2p3.fr
}

The contribution of the new results on $\Delta \alpha_{\text {had }}^{(5)}\left(m_{Z}^{2}\right)$ is now obtained by direct integration between measured KLOE and CMD-2 data points separately. For CMD-2 we use the "bare" cross section and for KLOE the pion form factor data. These are quantities in which the vacuum polarization corrections have been removed. The small $\rho$ contribution from lower and higher energies, not covered by new data, is evaluated as previously using the CMD-2 parametrization of the pion form factor. We treat the systematic uncertainties as fully correlated between different c.m. energies within the CMD-2 experiment. For the integration of the KLOE data, we constructed a covariance matrix based on the statistical covariance matrix with the addition of fully correlated systematic uncertainties as provided by the KLOE collaboration.

The results obtained from the dispersion integration of the KLOE and CMD-2 data at $m_{Z}^{2}$ are in good agreement with each other. The systematic uncertainty of the CMD-2 integration $(0.6 \%)$ is smaller than the corresponding uncertainty of the KLOE integration (1.4\%). On the other hand, the statistical uncertainty of the CMD-2 integration is slightly larger than the systematic one, while the statistical uncertainty of the KLOE integration is negligible. The integration results are combined as independent measurements in the evaluation of the $\rho$ contribution to $\Delta \alpha_{\text {had }}^{(5)}\left(m_{Z}^{2}\right)$.

We obtain a value of the hadronic contribution to the running of the QED fine structure constant of $\Delta \alpha_{\text {had }}^{(5)}(s)=$ $0.02758 \pm 0.00035$ at $s=m_{Z}^{2}$ corresponding to $1 / \alpha^{(5)}\left(m_{Z}^{2}\right)=128.940 \pm 0.048$. The value of the $\rho$ contribution has changed from 0.00350 in [1] to 0.00347 and the relative uncertainty has decreased from $2.3 \%$ to $0.9 \%$. The change of the uncertainty corresponds to the change of precision from the preliminary CMD-2 [2] data to the combination of published CMD-2 [4] and KLOE [6] data. The change of the value and the uncertainty of the hadronic contribution to the running of the QED fine structure constant at $m_{Z}^{2}$ is very small. In fact the $\rho$ region contributes to less than $13 \%$ to the dispersion integral and is known to much better precision than many of the other energy domains as can be concluded from Table I, which is updated from Ref. [1]. 
TABLE I. Contributions to $\Delta \alpha_{\text {had }}^{(5)}\left(m_{Z}^{2}\right)$.

\begin{tabular}{lcc}
\hline Range $\sqrt{s}, \mathrm{GeV}$ & $\Delta \alpha$ & Relative error \\
\hline$\rho$ & 0.00347 & $0.9 \%$ \\
Narrow resonances & 0.00184 & $3.1 \%$ \\
$1.05-2.0$ & 0.00156 & $15 \%$ \\
$2.0-5.0$ & 0.00381 & $5.9 \%$ \\
$5-7$ & 0.00183 & $6 \%$ \\
$7-12$ & 0.00304 & $1.4 \%$ \\
$>12$ & 0.01203 & $0.2 \%$ \\
\hline Total & 0.02758 & $1.3 \%$ \\
\hline
\end{tabular}

We note however, that the shape of the hadronic cross sections measured by the KLOE and CMD-2 collaborations differ for some individual points by more than the systematic uncertainty would indicate [7]. There also appears to be a small but systematic energy shift in the observed cross sections between the KLOE and the CMD-2 data, which at present is not understood. The effect on the integrated cross sections which contribute to $\Delta \alpha_{\text {had }}^{(5)}\left(m_{Z}^{2}\right)$ is however negligible.

The situation is different for the hadronic contribution to the anomalous magnetic moment of the muon $(g-2)_{\mu}[8-$ 11]. There the $\rho$ region provides the dominant contribution. The dispersion integral involves a different kernel which gives more weight to lower energies and larger sensitivity on systematic energy shifts.

Figure 1 (updated from Ref. [1]) gives the summary of $R_{\text {had }}$ measurements by different experiments and the current precision in different $e^{+} e^{-}$center-of-mass energy regions. $R_{\text {had }}$ is the measured QED cross section of the process $e^{+} e^{-} \rightarrow$ hadrons, normalized to the QED cross section for lepton-pair production. The uncertainty in the $1-2 \mathrm{GeV}$ energy region is $15 \%$. This region contributes to about $40 \%$ to the uncertainty on dispersion integral at $m_{Z}^{2}$, as can be seen from Table 1 and Fig. 2 in Ref. [1]. We

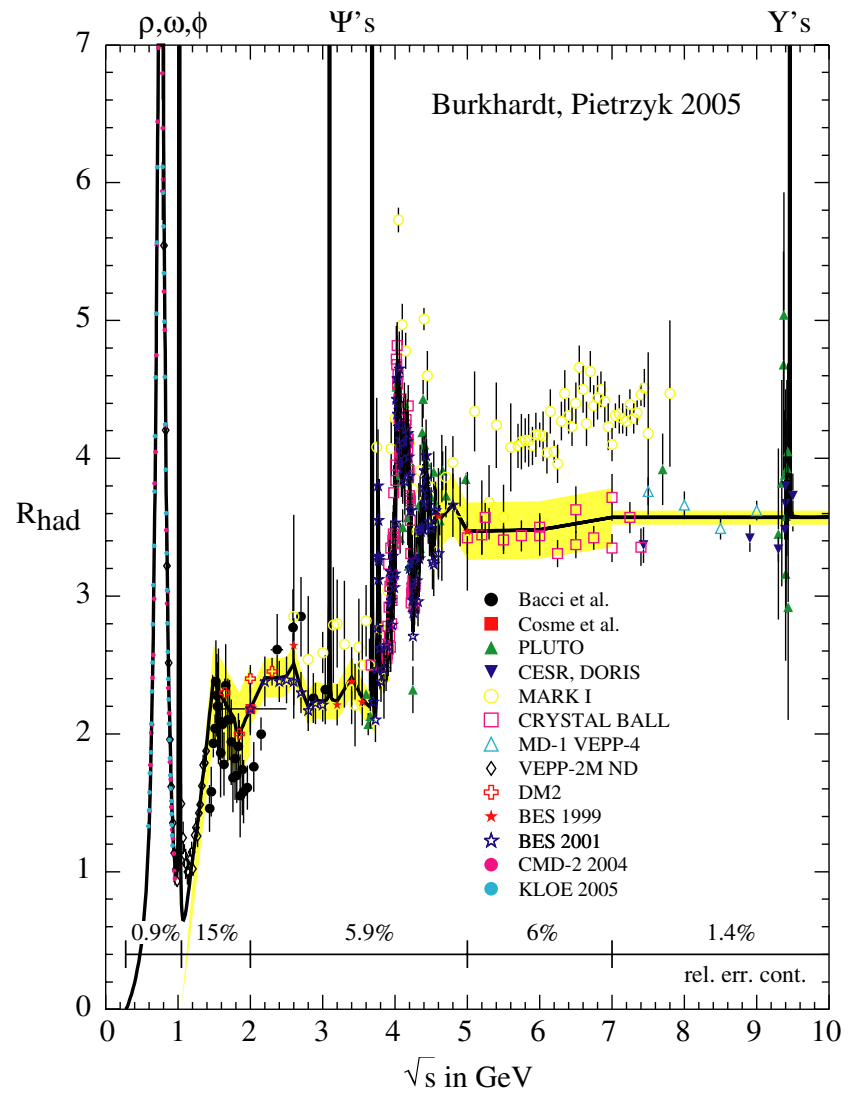

FIG. 1 (color online). $R_{\text {had }}$ including resonances. Measurements are shown with statistical errors. The relative uncertainty assigned to our parametrization is shown as band and given with numbers at the bottom.

would like to strongly encourage efforts to measure precisely $R_{\text {had }}$ in this c.m. energy region.

We would like to thank S. I. Eidelman from the CMD-2 Collaboration and A. Denig and G. Venanzoni from the KLOE Collaboration for useful discussions.
[1] H. Burkhardt and B. Pietrzyk, Phys. Lett. B 513, 46 (2001).

[2] R. R. Akhmetshin et al. (CMD-2 Collaboration), hep-ex/ 9904027.

[3] R. R. Akhmetshin et al. (CMD-2 Collaboration), Phys. Lett. B 527, 161 (2002).

[4] R. R. Akhmetshin et al. (CMD-2 Collaboration), Phys. Lett. B 578, 285 (2004).

[5] B. Pietrzyk and H. Burkhardt, Report No. LAPP-EXP 2003-07, 2003
[6] A. Aloisio et al., Phys. Lett. B 606, 12 (2005).

[7] See, for example, G. Venanzoni and F. Teubert, "Proceedings of the 32nd International Conference on HEP, Beijing, 2004.'

[8] M. Davier, S. Eidelman, A. Hoecker, and Z. Zhang, Eur. Phys. J. C 31, 503 (2003).

[9] F. Jegerlehner, Nucl. Phys. B Proc. Suppl. 126, 325 (2004); 131, 213 (2004).

[10] T. Teubner, Nucl. Phys. B Proc. Suppl. 131, 201 (2004).

[11] M. Davier, Nucl. Phys. B Proc. Suppl. 144, 250 (2005). 